\title{
NONLINEAR SIGNAL RECONSTRUCTION BASED ON THE DECOMPOSITION INTO CHAOTIC COMPONENTS
}

\author{
A. S. Sheludko, South Ural State University, Chelyabinsk, Russian Federation, \\ sheludkoas@susu.ru.
}

The paper proposes a signal reconstruction technique based on the decomposition into chaotic components. The considered approach can be usefully associated with the filtering, forecasting and control algorithms when only a small number of data samples is available. The developed decomposition algorithm involves sequential component extraction and recursive computation of the cost function. Some related questions are also discussed: choice of the class of chaotic maps, computational complexity of parameter estimation.

Keywords: signal reconstruction, chaotic map, parameter estimation, multiextremal cost function.

\section{Introduction}

Chaos and nonlinear dynamics provide modeling techniques [1, 2] for different applications [3]. Consider the problem of signal reconstruction from a small number of data samples. One of the most common approaches is decomposition into additive components $[4,5]$. In this paper chaotic maps [6] are used as models of the components. This approach can improve the accuracy of state estimation [7] and short-term forecasting [8].

The model of data signal. The data signal is reconstructed as a linear combination of chaotic components:

$$
y_{k}=\sum_{i=1}^{m} a_{i} x_{i, k}+a_{m+1}+v_{m, k}, \quad k=1,2, \ldots, N,
$$

where $y_{k}$ - data signal; $x_{i, k}, i=1,2, \ldots, m$ - chaotic components; $v_{m, k}$ - approximation errors; $a_{i}, i=1,2, \ldots, m+1$ - weight coefficients; $i$ - component index; $k$ - time step; $m$ - number of components; $N$ - number of data samples.

The components $x_{i, k}, i=1,2, \ldots, m$ are given by chaotic maps:

$$
x_{i, k}=f\left(x_{i, k-1}, \lambda_{i}\right), i=1,2, \ldots, m,
$$

where $\lambda_{i}, i=1,2, \ldots, m$ are parameters of the maps. The values of the variables $x_{i, k}$, $i=1,2, \ldots, m$ at the time step $k$ depends on the initial conditions $x_{i, 0}, i=1,2, \ldots, m$ :

$$
x_{i, k}=f^{k}\left(x_{i, 0}, \lambda_{i}\right), i=1,2, \ldots, m .
$$

Notation $f^{k}$ denotes $k$ iterations of the map $f$ :

$$
f^{k}\left(x_{0}, \lambda\right)=\underbrace{f(f(f \ldots f}_{k}\left(x_{0}, \lambda\right) \ldots))
$$

The problem is to estimate the weight coefficients $a_{i}, i=1,2, \ldots, m+1$, initial conditions $x_{i, 0}, i=1,2, \ldots, m$ and parameters $\lambda_{i}, i=1,2, \ldots, m$. 
Choice of the class of chaotic maps. A one-dimensional map $f: X \rightarrow X$ is chaotic on $X$ if $f$ satisfies the following conditions [9]:

1. $f$ has sensitive dependence on initial conditions: there exists $\delta>0$ such that, for any $x \in X$ and any $\epsilon>0$, there exists $y \in X$ such that $|x-y|<\epsilon$ and $\left|f^{k}(x)-f^{k}(y)\right| \geq \delta$ for some $k>0$.

2. $f$ is topologically transitive: for any pair of open sets $U, V \subseteq X$ there exists $k>0$ such that $f^{k}(U) \cap V \neq \oslash$.

3. Periodic points of the map $f$ are dense in $X$ : there exists at least one periodic point in any neighborhood of any $x \in X$.

The choice of the class of chaotic maps (2) depends on the characteristics of the data signal $y_{k}$, such as fractal dimension [10] and autocorrelation function [11]. Different classes can be defined by the sufficient conditions given in [12]. In [13], one-parameter families of chaotic maps are defined as ratios of polynomials. A new class of maps (Vertigo family), which are extensions of chaotic maps on the unit interval, is introduced in [14]. Chaotic signals passed through linear filters [15] can also be used as a components of the linear combination (1).

\section{The Algorithm of Parameter Estimation}

The proposed algorithm is based on sequential component extraction, i.e. sequential reconstruction of the component models (2). Consider the estimation of the initial condition $x_{m, 0}$ and parameter $\lambda_{m}$ for the component $x_{m, k}$. It is assumed that the estimates $\hat{x}_{i, 0}, \hat{\lambda}_{i}, i=1,2, \ldots, m-1$ have been already obtained. Estimates $\hat{x}_{m, 0}, \hat{\lambda}_{m}$ are obtained via minimization of the cost function

$$
F_{N}\left(x_{m, 0}, \lambda_{m}\right)=\sum_{k=1}^{N} \theta_{k}\left(y_{k}-\mathbf{G}_{k}\left(x_{m, 0}, \lambda_{m}\right) \mathbf{c}_{k}\right)^{2} .
$$

The matrix $\mathbf{G}_{k}$ consists of the values $x_{i, k}, i=1,2, \ldots, m$ at the time step $k$ :

$$
\mathbf{G}_{k}\left(x_{m, 0}, \lambda_{m}\right)=\left[\begin{array}{lllll}
x_{1, k} & \ldots & x_{m-1, k} & x_{m, k}\left(x_{m, 0}, \lambda_{m}\right) & 1
\end{array}\right] .
$$

Notation $x_{m, k}\left(x_{m, 0}, \lambda_{m}\right)$ means that the variable $x_{m, k}$ depends on the cost function arguments (initial condition $x_{m, 0}$ and parameter $\lambda_{m}$ ):

$$
x_{m, k}=f^{k}\left(x_{m, 0}, \lambda_{m}\right) .
$$

The elements $c_{i, k}, i=1,2, \ldots, m+1$ of the coefficient vector

$$
\mathbf{c}_{k}=\left[\begin{array}{llll}
c_{1, k} & \ldots & c_{m, k} & c_{m+1, k}
\end{array}\right]^{\mathrm{T}}
$$

correspond to the weight coefficients $a_{i}, i=1,2, \ldots, m+1$ of the linear combination (1). For certain values of the initial condition $x_{m, 0}$ and parameter $\lambda_{m}$, the coefficient vector $\mathbf{c}_{k}$ is calculated recursively:

$$
\begin{gathered}
\mathbf{c}_{k}=\mathbf{c}_{k-1}+\mathbf{L}_{k}\left(y_{k}-\mathbf{G}_{k} \mathbf{c}_{k-1}\right), \\
\mathbf{L}_{k}=\mathbf{P}_{k-1} \mathbf{G}_{k}^{\mathrm{T}}\left(\mathbf{G}_{k} \mathbf{P}_{k-1} \mathbf{G}_{k}^{\mathrm{T}}+r_{m}\right)^{-1}, \\
\mathbf{P}_{k}=\left(\mathbf{I}-\mathbf{L}_{k} \mathbf{G}_{k}\right) \mathbf{P}_{k-1},
\end{gathered}
$$


where $\mathbf{I}$ is the identity matrix of dimension $(m+1) \times(m+1) ; r_{m}$ is the prior estimate of the variance of the approximation errors $v_{m, k}$. Equations (4)-(6) represent the recursive least square estimation [16]. It is also a special case of the Kalman filter applied to the state-space model

$$
\mathbf{c}_{k}=\mathbf{c}_{k-1}, y_{k}=\mathbf{G}_{k} \mathbf{c}_{k}+v_{m, k} .
$$

In this interpretation, the variables $\mathbf{c}_{k}, \mathbf{G}_{k}, \mathbf{L}_{k}$ and $\mathbf{P}_{k}$ are the state vector, measurement matrix, filter gain and estimation error covariance, respectively.

Recursive computation assumes that the accuracy of the value of $\mathbf{c}_{k}$ increases with the number of time steps. Therefore the cost function (3) is weighted by the coefficients $\theta_{k} \in[0,1]:$

$$
\theta_{k}=1-\mathbf{e}^{-\frac{\beta}{k}}
$$

where $\beta>0$. Due to the recursive form of (4)-(6), it is also reasonable to calculate the cost function (3) recursively:

$$
F_{k}=F_{k-1}+\theta_{k}\left(y_{k}-\mathbf{G}_{k} \mathbf{c}_{k}\right)^{2},
$$

where $F_{0}=0$. Minimization of the cost function (3) provides estimates $\hat{x}_{m, 0}, \hat{\lambda}_{m}$ for the component $x_{m, k}$. Then the weight coefficients of the linear combination (1) are updated:

$$
\hat{a}_{i}=c_{i, N}, i=1,2, \ldots, m+1,
$$

where $c_{i, N}, i=1,2, \ldots, m+1$ are obtained via $(4)-(6)$ for the found estimates $\hat{x}_{m, 0}, \hat{\lambda}_{m}$ of the initial condition $x_{m, 0}$ and parameter $\lambda_{m}$.

\section{Numerical Example}

Consider the data signal produced by the Henon map [6]

$$
y_{k}=1-\alpha_{1} y_{k-1}^{2}+\alpha_{2} y_{k-2},
$$

which is chaotic for $\alpha_{1}=1.4, \alpha_{2}=0.3$. The initial conditions $y_{0}=0, y_{1}=0.5$, the number of data samples $N=30$. The logistic maps are used as models of the components $x_{i, k}$, $i=1,2, \ldots, m$ :

$$
x_{i, k}=\lambda_{i} x_{i, k-1}\left(1-x_{i, k-1}\right), i=1,2, \ldots, m .
$$

If $m=1$ the initial value of the coefficient vector is set to zero: $\mathbf{c}_{0}=\left[\begin{array}{ll}0 & 0\end{array}\right]^{\mathrm{T}}$. If $m>1$ the previously obtained estimates are used to set the initial value:

$$
\mathbf{c}_{0}=\left[\begin{array}{lllll}
\hat{a}_{1} & \ldots & \hat{a}_{m-1} & 0 & \hat{a}_{m}
\end{array}\right]^{\mathrm{T}} .
$$

The prior estimate of the variance of the approximation errors

$$
r_{m}=\left\{\begin{array}{r}
\left(\frac{\sigma_{y}}{2}\right)^{2}, m=1, \\
\left(\frac{\sigma_{v_{m-1}}}{2}\right)^{2}, m>1,
\end{array}\right.
$$

where $\sigma_{y}, \sigma_{v_{m-1}}$ are the sample standard deviations. Thus, it is assumed that the extraction of the component $x_{m, k}$ reduce the standard deviation of the approximation errors $v_{m, k}$ by a factor of 2 . 
Particle Swarm Optimization (PSO) $[17,18,19]$ is applied to find the global minimum of the cost function (3). In (7) parameter $\beta=10$. Fig. 1, 2 show the multiextremal cost function $F_{N}\left(x_{1,0}, \lambda_{1}\right)$ that corresponds to the reconstruction of the first component $x_{1, k}$. The set of 100 particles is used to find the global minimum. Fig. 3 shows the final positions of the particles after 500 iterations of the PSO.

Finally, the data signal $y_{k}$ is reconstructed as the linear combination of $m=3$ components (Fig. 5). The estimated parameters of the model (1), (8) are given in Table. Fig. 4 shows the recursive computation of the weight coefficients.

Table

Estimates of the model parameters

\begin{tabular}{|c|c|c|c|}
\hline$i$ & $\hat{\lambda}_{i}$ & $\hat{x}_{i, 0}$ & $\hat{a}_{i}$ \\
\hline 1 & 3.71597947 & 0.28017384 & 3.46155612 \\
\hline 2 & 3.76572989 & 0.24220516 & 0.81497019 \\
\hline 3 & 3.82845562 & 0.24131992 & -0.32950922 \\
\hline 4 & - & - & -2.27867173 \\
\hline
\end{tabular}

In order to estimate the quality of the approximation, consider $n=100$ examples with different initial conditions $y_{0} \in[-0.25,0.25], y_{1} \in[-1,1]$. The values of $y_{0}, y_{1}$ are generated by the function rand of Matlab. The relative error of the approximation is calculated as follows:

$$
\epsilon_{m}=\frac{\sigma_{v_{m}}}{\sigma_{y}} \times 100 \%
$$

where $\sigma_{v_{m}}, \sigma_{y}$ are the sample standard deviations of the approximation errors $v_{m, k}$ and data signal $y_{k}$, respectively. Fig. 6 shows the dependence of the average relative error $\epsilon_{m}$ on the number $m$ of components.

\section{Conclusion}

The proposed algorithm is based on sequential component extraction. The extraction of each component requires the minimization of the cost function that depends only on two variables: the initial condition and parameter of chaotic map. The weight coefficients of the linear combination are calculated recursively together with the value of the cost function. The main difficulty is that the cost function becomes extremely complex and has a large number of local minima. Thus, it is necessary to use global optimization algorithms. At the same time, nonlinear properties of chaotic maps can provide the required accuracy even for a small number of components.

The work was supported by Act 211 Government of the Russian Federation, contract № 02.A03.21.0011. 


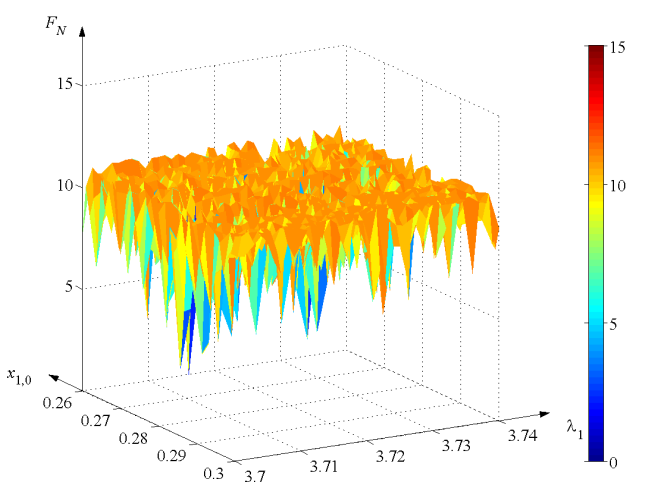

Fig. 1. Multiextremal cost function

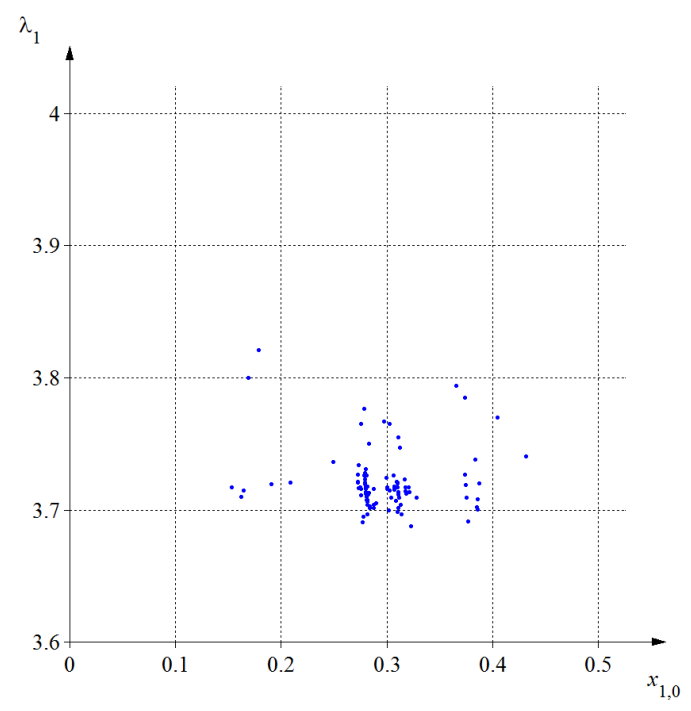

Fig. 3. Final positions of the particles after 500 iterations of the PSO

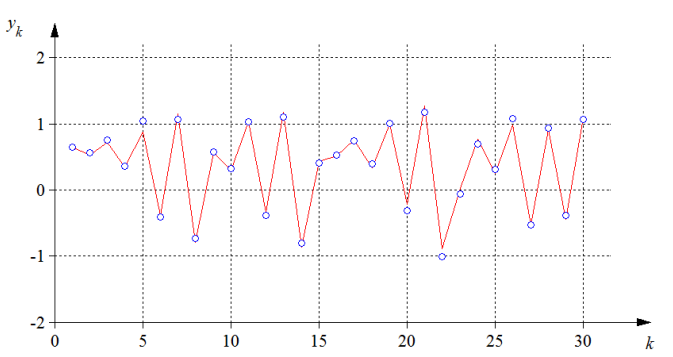

Fig. 5. Approximation of the data signal for $m=3$ (points are the true values)

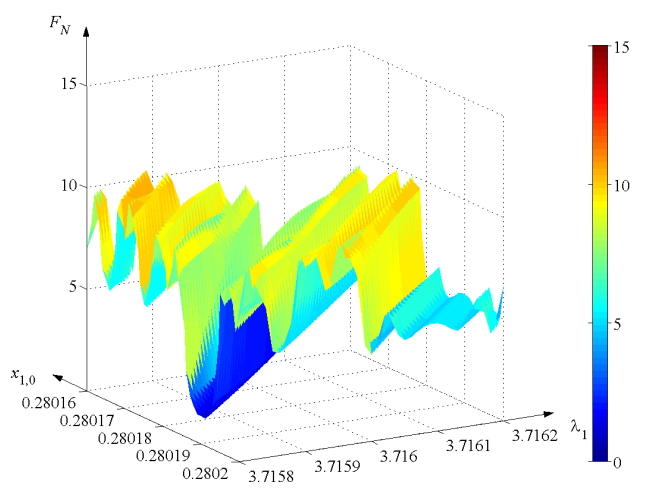

Fig. 2. Cost function near the global minimum

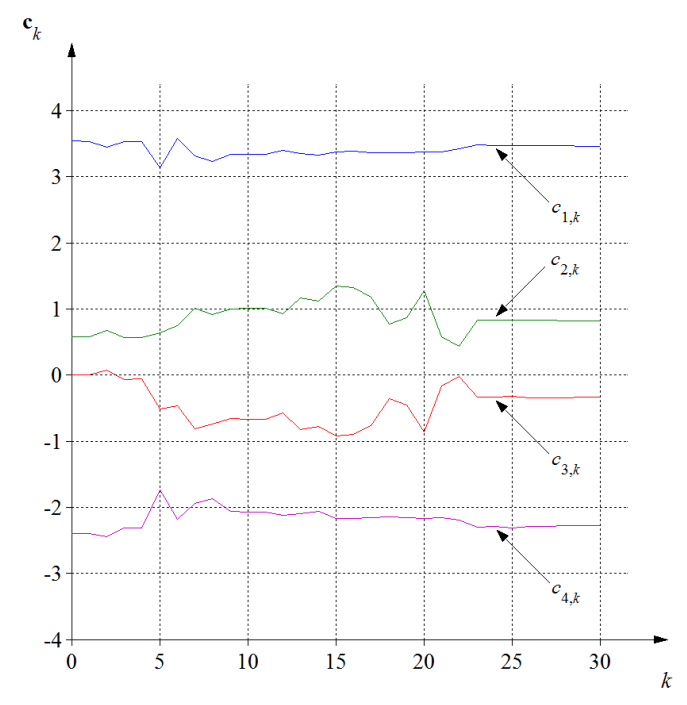

Fig. 4. Recursive computation of the coefficient vector $\mathbf{c}_{k}$

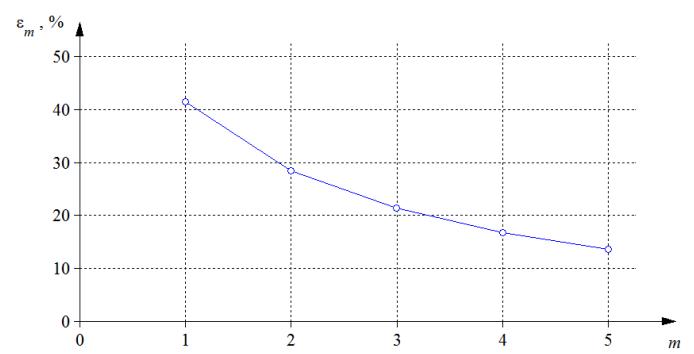

Fig. 6. Dependence of the averaged relative error $\epsilon_{m}$ on the number $m$ of components 


\section{References}

1. Aguirre L.A., Letellier C. Modeling Nonlinear Dynamics and Chaos: A Review. Mathematical Problems in Engineering, 2009, vol. 2009. doi: 10.1155/2009/238960.

2. Bezruchko B.P., Smirnov D.A. Extracting Knowledge From Time Series. An Introduction to Nonlinear Empirical Modeling. Springer, Berlin, Heidelberg, 2010. doi: 10.1007/978-3-642-12601-7.

3. Thompson J.M.T. Chaos, Fractals and Their Applications. International Journal of Bifurcation and Chaos, 2016, vol. 26, no. 13. doi: 10.1142/S0218127416300354.

4. Vitanov N.K., Sakai K., Dimitrova Z.I. SSA, PCA, TDPSC, ACFA: Useful Combination of Methods for Analysis of Short and Nonstationary Time Series. Chaos, Solitons \&J Fractals, 2008, vol. 37, no. 1, pp. 187-202. doi: 10.1016/j.chaos.2006.08.043.

5. Ljung L. Perspectives on System Identification. Annual Reviews in Control, 2007, vol. 34, issue 1, pp. 1-12. doi: 10.1016/j.arcontrol.2009.12.001.

6. Sprott J.C. Chaos and Time-Series Analysis. Oxford University Press, 2003.

7. Shiryaev V.I., Podivilova E.O. Set-valued Estimation of Linear Dynamical System State When Disturbance is Decomposed as a System of Functions. Procedia Engineering, 2015, vol. 129, pp. 252-258. doi: 10.1016/j.proeng.2015.12.045.

8. Malyutina E.I., Shiryaev V.I. Time Series Forecasting Using Nonlinear Dynamic Methods and Identification of Deterministic Chaos. Procedia Computer Science, 2014, vol. 31, pp. 1022-1031. doi: 10.1016/j.procs.2014.05.355.

9. Devaney R.L. An Introduction to Chaotic Dynamical Systems. Westview Press, 1989.

10. Dubovikov M.M., Starchenko N.V., Dubovikov M.S. Dimension of the Minimal Cover and Fractal Analysis of Time Series. Physica A, 2004, vol. 339, pp. 591-608. doi: 10.1016/j.physa.2004.03.025.

11. Anishchenko V.S., Vadivasova T.E., Okrokvertskhov G.A., Strelkova G.I. Correlation Analysis of Dynamical Chaos. Physica A, 2003, vol. 325, pp. 199-212. doi: 10.1016/S0378-4371(03)00199-7.

12. Erramilli A., Singh R.P., Pruthi P. An Application of Deterministic Chaotic Maps to Model Packet Traffic. Queueing Systems, 1995, vol. 20, pp. 171-206.

13. Jafarizadeh M.A., Behnia S., Khorram S., Nagshara H. Hierarchy of Chaotic Maps with an Invariant Measure. Journal of Statistical Physics, 2001, vol. 104, pp. 1013-1028.

14. Jafari S., Pham V.-T., Golpayegani S.M.R.H. et al. The Relationship Between Chaotic Maps and Some Chaotic Systems with Hidden Attractors. International Journal of Bifurcation and Chaos, 2016, vol. 26, no. 13. doi: 10.1142/S0218127416502114. 
15. Kipchatov A.A., Kozlenko E.L. Reconstruction of Chaotic Oscillations After Passage Through Linear Filters. Technical Physics Letters, 1999, vol. 25, no. 2, pp. 148-150. doi: $10.1134 / 1.1262382$.

16. Simon D. Optimal State Estimation: Kalman, H-infinity, and Nonlinear Approaches. Hoboken, NJ: Wiley, 2006.

17. Banerjee A., Abu-Mahfouz I. A Comparative Analysis of Particle Swarm Optimization and Differential Evolution Algorithms for Parameter Estimation in Nonlinear Dynamic Systems. Chaos, Solitons \& Fractals, 2014, vol. 58, pp. 65-83. doi: 10.1016/j.chaos.2013.11.004.

18. Jiang Y., Lau F.C.M., Wang S., Tse C.K. Parameter Identification of Chaotic Systems by a Novel Dual Particle Swarm Optimization. International Journal of Bifurcation and Chaos, 2016, vol. 26, no. 2. doi: 10.1142/S0218127416500243.

19. Gotmare A., Bhattacharjee S.S., Patidar R., George N.V. Swarm and Evolutionary Computing Algorithms for System Identification and Filter Design: A Comprehensive Review. Swarm and Evolutionary Computation, 2017, vol. 32, pp. 68-84. doi: 10.1016/j.swevo.2016.06.007.

Anton S. Sheludko, Department of Applied Mathematics and Programming, South Ural State University (Chelyabinsk, Russian Federation), sheludkoas@susu.ru

Received October 23, 2017

УДК 519.7

DOI: $10.14529 /$ jcem 170403

\section{РЕКОНСТРУКЦИЯ НЕЛИНЕЙНОЙ МОДЕЛИ СИГНАЛА В ВИДЕ РАЗЛОЖЕНИЯ ПО СИСТЕМЕ ХАОТИЧЕСКИХ ПРОЦЕССОВ}

\section{A.C. Шелудъко}

\footnotetext{
Для построения нелинейной модели сигнала предлагается использовать разложение по системе хаотических процессов. Рассматриваемый подход ориентирован на применение в алгоритмах фильтрации, прогнозирования и управления в условиях, когда доступно небольшое число измерений. Разрабатываемый алгоритм разложения использует последовательное выделение составляющих и рекуррентное вычисление значения целевой функции. Рассмотрены вопросы выбора класса хаотических отображений, сложность задачи оценивания параметров модели, связанная с многоэкстремальностью целевой функции.

Ключевые слова: реконструкиия сигнала, хаотическое отображение, оценивание параметров, многоэкстремальная целевая функиия.
} 


\section{Литература}

1. Aguirre, L.A. Modeling Nonlinear Dynamics and Chaos: A Review / L.A. Aguirre, C. Letellier // Mathematical Problems in Engineering. - 2009. - V. 2009.

2. Bezruchko, B.P. Extracting Knowledge From Time Series. An Introduction to Nonlinear Empirical Modeling / B.P. Bezruchko, D.A. Smirnov. - Berlin, Heidelberg: Springer, 2010.

3. Thompson, J.M.T. Chaos, Fractals and Their Applications / J.M.T. Thompson // International Journal of Bifurcation and Chaos. - 2016. - V. 26, № 13.

4. Vitanov, N.K. SSA, PCA, TDPSC, ACFA: Useful Combination of Methods for Analysis of Short and Nonstationary Time Series / N.K. Vitanov, K. Sakai, Z.I. Dimitrova // Chaos, Solitons \& Fractals. - 2008. - V. 37, № 1. - P. 187-202.

5. Ljung, L. Perspectives on System Identification / L. Ljung // Annual Reviews in Control. -2010 . - V. 34, is. 1. - P. 1-12.

6. Sprott, J.C. Chaos and Time-Series Analysis / J.C. Sprott. - Oxford University Press, 2003.

7. Shiryaev, V.I. Set-valued Estimation of Linear Dynamical System State When Disturbance is Decomposed as a System of Functions / V.I. Shiryaev, E.O. Podivilova // Procedia Engineering. - 2015. - V. 129. - P. 252-258.

8. Malyutina, E.I. Time Series Forecasting Using Nonlinear Dynamic Methods and Identification of Deterministic Chaos / E.I. Malyutina, V.I. Shiryaev // Procedia Computer Science. - 2014. - V. 31. - P. 1022-1031.

9. Devaney, R.L. An Introduction to Chaotic Dynamical Systems / R.L. Devaney. Westview Press, 1989.

10. Dubovikov, M.M. Dimension of the Minimal Cover and Fractal Analysis of Time Series / M.M. Dubovikov, N.V. Starchenko, M.S. Dubovikov // Physica A. - 2004. V. 339. - P. 591-608.

11. Anishchenko, V.S. Correlation Analysis of Dynamical Chaos / V.S. Anishchenko, T.E. Vadivasova, G.A. Okrokvertskhov, G.I. Strelkova // Physica A. - 2003. - V. 325.

- P. 199-212.

12. Erramilli, A. An Application of Deterministic Chaotic Maps to Model Packet Traffic / A. Erramilli, R.P. Singh, P. Pruthi // Queueing Systems. - 1995. - V. 20. - P. 171-206.

13. Jafarizadeh, M.A. Hierarchy of Chaotic Maps with an Invariant Measure M.A. Jafarizadeh, S. Behnia, S. Khorram, H. Nagshara // Journal of Statistical Physics. - 2001. - V. 104. - P. 1013-1028.

14. Jafari, S. he Relationship Between Chaotic Maps and Some Chaotic Systems with Hidden Attractors / S. Jafari, V.-T. Pham, S.M.R.H. Golpayegani et al. // International Journal of Bifurcation and Chaos. - 2016. - V. 26, № 13. 
15. Kipchatov, A.A. Reconstruction of Chaotic Oscillations After Passage Through Linear Filters / A.A. Kipchatov, E.L. Kozlenko // Technical Physics Letters. - 1999. - V. 25, № 2. - P. 148-150.

16. Simon, D. Optimal State Estimation: Kalman, H-infinity, and Nonlinear Approaches / D. Simon. - Hoboken, NJ: Wiley, 2006.

17. Banerjee, A. A Comparative Analysis of Particle Swarm Optimization and Differential Evolution Algorithms for Parameter Estimation in Nonlinear Dynamic Systems / A. Banerjee, I. Abu-Mahfouz // Chaos, Solitons \& Fractals. - 2014. - V. 58. - P. 65-83.

18. Jiang, Y. Parameter Identification of Chaotic Systems by a Novel Dual Particle Swarm Optimization / Y. Jiang, F.C.M. Lau, S. Wang, C.K. Tse // International Journal of Bifurcation and Chaos. - 2016. - V. 26, № 2.

19. Gotmare, A. Swarm and Evolutionary Computing Algorithms for System Identification and Filter Design: A Comprehensive Review / A. Gotmare, S.S. Bhattacharjee, R. Patidar, N.V. George // Swarm and Evolutionary Computation. - 2017. - V. 32. - P. 68-84.

Шелудъко Антон Сергеевич, кафедра прикладной математики и программирования, Южно-Уральский государственный университет (ә. Челябинск, Российская Федераu, я), sheludkoas@susu.ru

Поступила в редакцию 23 октября 2017 г. 